\title{
DISSECTION TECHNIQUE FOR THE STUDY OF THE CEREBRAL SULCI, GYRI AND VENTRICLES
}

\author{
João Paulo Mattos', Marcos Juliano dos Santos' João Flavio Daniel Zullo², \\ Andrei Fernandes Joaquim ${ }^{2}$, Feres Chaddad-Neto', Evandro de Oliveira ${ }^{3}$
}

\begin{abstract}
Neuroanatomy in addition to neurophysiology, are the basic areas for the proper formation from health students to specialized professionals in neuroscience. A step by step guide for practical studies of neuroanatomy is required for this kind of knowledge to become more acceptable among medical students, neurosurgeons, neurologists, neuropediatricians and psychiatric physicians. Based on the well known courses of sulci, gyri and ventricles offered by Beneficência Portuguesa Hospital in São Paulo, Brazil, two times a year, since 1994, totalizing more than 20 complete courses, and answering the request of many neuroscience students and professionals whose asked for a practical guide to the neuroanatomy study, the authors suggest a protocol for the study of superficial and deep brain structures showing how to approach the more structures as possible with minimum damage to the anatomic piece and with the smaller number of brains.
\end{abstract}

KEY WORDS: neuroanatomy, brain, dissection technique.

\section{Técnica de dissecação para o estudo dos sulcos, giros e ventriculos cerebrais}

Resumo - Neuroanatomia e a neurofisiologia são as áreas básicas para a adequada formação desde estudantes na área da saúde a profissionais especializados em neurociências. Um guia prático, passo a passo, para o estudo de neuroanatomia é necessário para tornar esse conhecimento mais acessivel entre estudantes de medicina, neurologistas, neurofisiologistas, neurocirurgiões, neuropediatras e psiquiatras. Baseados em cursos reconhecidos nacional e internacionalmente a respeito dos sulcos, giros e ventrículos cerebrais elaborados e realizados pelo Instituto de Ciências Neurológicas (ICNE) no Hospital de Beneficência Portuguesa de São Paulo, Brasil, duas vezes ao ano, totalizando mais de 20 cursos já realizados, os autores apresentam um protocolo de dissecação para o estudo das estruturas superficiais e profundas do encéfalo, mostrando como expor o máximo de estruturas com um mínimo de manipulação e dano à peça anatômica e utilizando o menor número de encéfalos possíveis.

PALAVRAS-CHAVE: neuroanatomia, cérebro, técnicas de dissecação.

The study of neuroanatomy is one of the most challenging issues for the proper formation of medical students, residents of neurology, neurosurgery, neuropediatrics and for those interested in medical sciences. Therefore, according to Brazilian Ministry of Education and Culture, there are terms for all medical schools that make the study of neuroanatomy an obligation for the acceptance of that institution as one registered and allowed to offer neuroscience residency programs'. The use of fixed brains for this purpose is wide accepted but some details of neuroanatomy are usually missed if a defined protocol of dissection is not followed. The damage to the formolized brain is another matter that can happen if there is no guidance in that study, especially with the difficult access to these organs in most places.

The description of the already namely structures, sul$\mathrm{ci}$, gyri and ventricles elements and its relations are not the objective of this paper as it is already described and wide accessible to all neuroscience students ${ }^{2-5}$.

We suggest a step by step protocol of brain dissection for the study and identification of the supratentorial structures since the acquisition of the brain from the cadaver to the study of the deep anatomic structures.

\section{METHOD}

The brains used in the courses mentioned before in the text,

\footnotetext{
Laboratório de Microcirurgia, Hospital Beneficência Portuguesa de São Paulo, São Paulo SP, Brasil; Instituto de Ciências Neurológicas, São Paulo SP, Brasil (ICNE) and Disciplina de Neurocirurgia, Faculdade de Ciências Médicas da Universidade de Campinas, Campinas SP, Brazil (UNICAMP): ${ }^{1}$ Médico Assistente; ${ }^{2}$ Médico Residente; ${ }^{3}$ Professor Doutor.
}

Received 14 December 2007, received in final form 17 March 2008. Accepted 3 April 2008.

Dr. Feres Chaddad Neto - Praça Amadeu Amaral 47 / 79 andar - 01327-010 São Paulo SP - Brasil. 
as well as those dissected and shown in the pictures comes from the Serviço de Verificação de Óbitos (SVO) from S.Paulo, Brazil, which is a public organ for death investigation. A fresh brain is the best way to achieve an adequate neuroanatomical study. It must be removed from the cadaver as soon as possible. The internal carotids and the basilar artery should be catheterized with a number 10 vesical catheter and washed with saline solution or water for several times, until the blood is washed away from the tissue and, after that, the brain should be immediately immersed in $4 \%$ formol solution. It must be kept immersed for a month, at least. After that, the fixated brain is washed with abundant water for beginning the steps for practical study.

\section{RESULTS}

\section{Step 1}

The arachnoid and the cortical and sulcal vessels must be taken out from the brain to expose the sulci and gyri. An adequate technique must be followed to avoid damage to the cortex as shown in Figure 1A. After that, the brain is ready to begin the identification of superficial and basal structures.

The brain has three surfaces: medial, superolateral and basal surfaces. They are limited by the borders of the brain: superomedial, inferolateral, medial occipital and medial orbital. The identification of the sulci and gyri begins from the convexity (Table), in the superolateral surface, which is limited by the superolateral and inferolateral borders. The sylvian fissure, or lateral sulcus, and its divisions must be identified first. The anterior horizontal ramus and anterior ascending ramus of the lateral sulcus must be looked for, especially in the left side, where they are the limits for the Broca's area. The posterior ramus of the lateral sulcus should be identified as well. The insula, in the floor of the sylvian fissure, and its relations with each division of this fissure is very important, and should be looked for ${ }^{2,3}$.

The superior (SFS) and inferior frontal sulci (IFS) as the superior (SFG), middle (MFG) and inferior (IFG) frontal gyri must be identified. At the left side, the 3 divisions of inferior frontal gyrus between the anterior ascending and anterior horizontal ramus of the lateral sulcus are found (pars orbitalis, triangularis and opercularis) and its correspondence with the insula limits must be searched for ${ }^{2-4,6-8}$. The relations of the superficial structures with the deep ones, as the place of foramen of Monro according to the superficial landmarks must always be looked for ${ }^{2-4,6-8}$. Precentral (PreCS), central (CS) and post-central sulci (PosCS) are also identified. The pre-central and pos-central gyri are identified with the CS between then, forming the "central lobe". The intra-parietal sulcus (IPS) coming from post-central or superior temporal sulcus (STS) are identified. It divides the parietal lobe in superior and inferior parietal lobules. The point where the IPS finds the PosCS
Table. Checklist for the study of the superficial brain structures.

\begin{tabular}{|c|c|}
\hline SUPEROLATERAL SURFACE & MEDIAL SURFACE \\
\hline Lateral sulcus (Sylvian fissure) & Rostrum of corpus callosum \\
\hline Stem along sphenoid ridge & Genu of corpus callosum \\
\hline Anterior horizontal ramus & Body of corpus callosum \\
\hline Anterior asceding ramus & Splenium of corpus callosum \\
\hline Posterior ramus & Septum pelucidum \\
\hline \multirow[t]{2}{*}{ Insula in the floor } & Paraterminal gyrus \\
\hline & Postolfactory sulcus \\
\hline \multirow[t]{2}{*}{ Central sulcus } & Anterior paraolfactoy sulcus \\
\hline & Subcallosal area \\
\hline Frontal lobe & Cingulate sulcus \\
\hline Precentral gyrus and sulcus & Medial frontal gyrus \\
\hline Superior frontal gyrus and sulcus & Paracentral lobule \\
\hline Middle frontal gyrus & Cingulate gyrus \\
\hline Inferior frontal gyrus and sulcus & Isthmus of the cingulate gyrus \\
\hline \multirow[t]{2}{*}{ Pars orbitalis,triangularis,opercularis } & Parahipoccampal gyrus \\
\hline & Parietocciptal sulcus \\
\hline Parietal lobe & Calcarine sulcus \\
\hline Central sulcus (anterior limit) & Cuneus \\
\hline Postcentral sulcus & Precuneus \\
\hline \multicolumn{2}{|l|}{ Postcentral gyrus } \\
\hline \multicolumn{2}{|l|}{ Intraparietal sulcus } \\
\hline Superior parietal lobule & INFERIOR SURFACE \\
\hline \multicolumn{2}{|l|}{ Inferior parietal lobule } \\
\hline Supramarginal gyrus & Anterior part (orbital) \\
\hline Angular gyrus & Olfactory sulcus \\
\hline \multirow[t]{2}{*}{ Arcus tempora-occiptales } & Gyrus rectus \\
\hline & Anterior orbital gyrus \\
\hline Temporal lobe & Posterior orbital gyrus \\
\hline Superior temporal sulcus & Medial orbital gyrus \\
\hline Superior temporal gyrus & Lateral orbital gyrus \\
\hline \multicolumn{2}{|l|}{ Inferior temporal sulcus } \\
\hline Inferior temporal gyrus & Posterior part \\
\hline \multirow[t]{2}{*}{ Lateral end of tranv. temporal gyri } & Collateral and occiptotemporal sulci \\
\hline & Rhinal sulcus \\
\hline Occiptal lobe & Lingula \\
\hline Preocciptal notch & Parahipoccampal gyrus \\
\hline Superior end of parietocciptal sulcus & Medial occiptotemporal gyrus \\
\hline Tranverse occiptal sulcus & Lateral occiptotemporal gyrus \\
\hline \multicolumn{2}{|l|}{ Lateral occiptal sulcus } \\
\hline \multicolumn{2}{|l|}{ Superior occiptal gyri } \\
\hline \multicolumn{2}{|l|}{ Inferior occiptal gyri } \\
\hline \multicolumn{2}{|l|}{ Insula } \\
\hline \multicolumn{2}{|l|}{ Circular sulcus } \\
\hline \multicolumn{2}{|l|}{ Central sulcus of insula } \\
\hline Long and short gyri of insula & \\
\hline
\end{tabular}

is called intra-parietal point and takes you to the atrium of ventricles ${ }^{9,10}$. The occipital lobe has an inconstant gyri and sulci but one lateral occipital sulci at least must be identified as continuity from the IPS. The limits of the occiptal lobe, given by anatomic landmarks should be recognized in superolateral surface ${ }^{2,11}$, a imaginary line between preocciptal notch and the superficial end of the parietocciptal sulcus is the anterior limit of this lobe. 


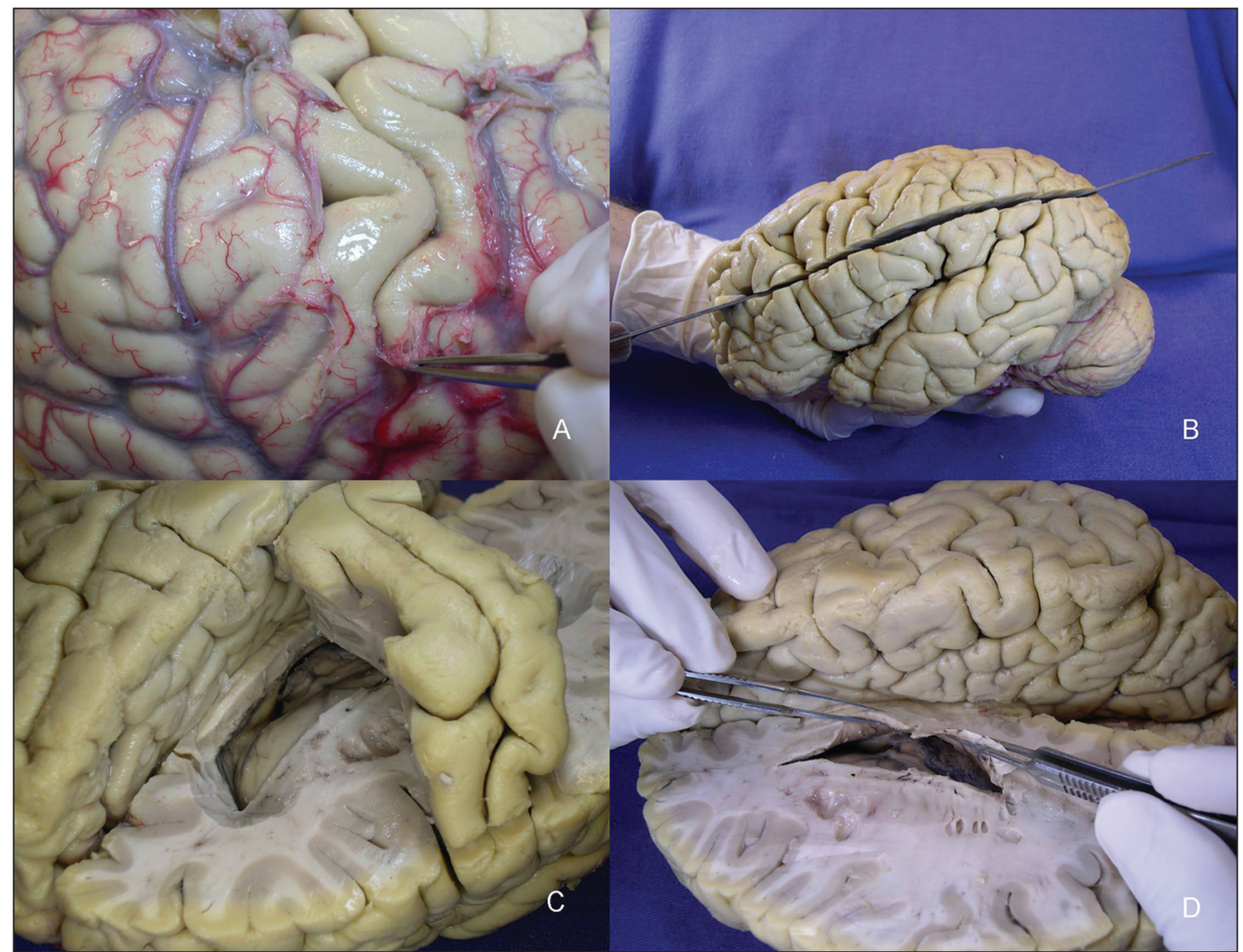

Fig 1. (A) After the fixed brain is achieved, the arachnoid membrane and its vessels must be taken off using appropriate technique. One of the easiest way is to hold the major sulcal arteries near the superior convexity border and pull it all the way down following the same direction of the sulci. It will avoid that strong and hard vessels cut the brain surface when pulled off. (B) As exposed earlier, the long knife is located in the frontoparietal operculum with the base of the pars triangularis and a point $2 \mathrm{~cm}$ superior to the posterior end of the sylvian fissure as landmarks. This cut must be aimed to the superior limit of the corpus callosum, in the midline, to preserve the lateral ventricle roof to be opened later, what can be difficult in some hemispheres. If that is the case, this step should be done with more than one movement, with deeper cuts until it gets in the corpus callosum by the midline. (C) The pre-central gyrus is separated from the remained frontal and parietal lobes and put in its original position for the study of the relations between the primary motor cortex with the deeper structures as the corpus callosum and foramen of Monro. In the picture, the poscentral gyrus is also placed in anatomic position, defining the "central lobe" and the paracentral lobule in the medial surface. This step can be done before and after the removing of the lateral ventricular roof, as shown in the picture. (D) Opening the lateral ventricular roof. With a number 10 blade, the roof of the lateral ventricle is removed, beginning from the frontal horn, in an anterior-posterior way. Care should be taken here, to avoid lesion of the ventricular floor. If the first cut in the hemisphere, made by the long-knife, was succesfull, the ventricular roof should be thin as 1$2 \mathrm{~mm}$, what makes easier the identification of the ventricular cavity to be opened. The ventricular cavity must be entirely exposed, from the frontal horn to the atrium in this step.

The basal surface is limited by the inferolateral and medial occipital and medial orbital borders of the brain. The basal sulci and gyri from temporal and frontal lobes are also studied $(\text { Table })^{6}$. The relation of anterior perforated substance with the neighborhood structures, cisterns and with the deep correlations in the basal ganglia is a step that should not be forgotten. Also, the basal cistern regions and its relation must be studied ${ }^{2,3,6,12}$.

In the medial surface, between the medial orbital and occiptal borders and the superolateral border of the brain, the portions of the corpus callosum, paraterminal gyrus, postolfatory sulcus, paraolfatory gyrus, cingulated sulcus, medial frontal gyrus, paracentral lobule, cingulated gyrus continuing through isthmus to parahippocampal gyrus, parietocciptal sulcus, calcarine sulcus, cuneus and precuneus as especified in Table ${ }^{2-4,6-8}$.

\section{Step 2}

After the identification of superficial structures, the brain must be cut to provide a deep brain structures study.

A long knife is used to perform this step. All its length must be used to do this step at once as demonstrated by Figure 1B. The anterior point to put the knife in place is the base of the pars triangularis of the IFG and the pos- 


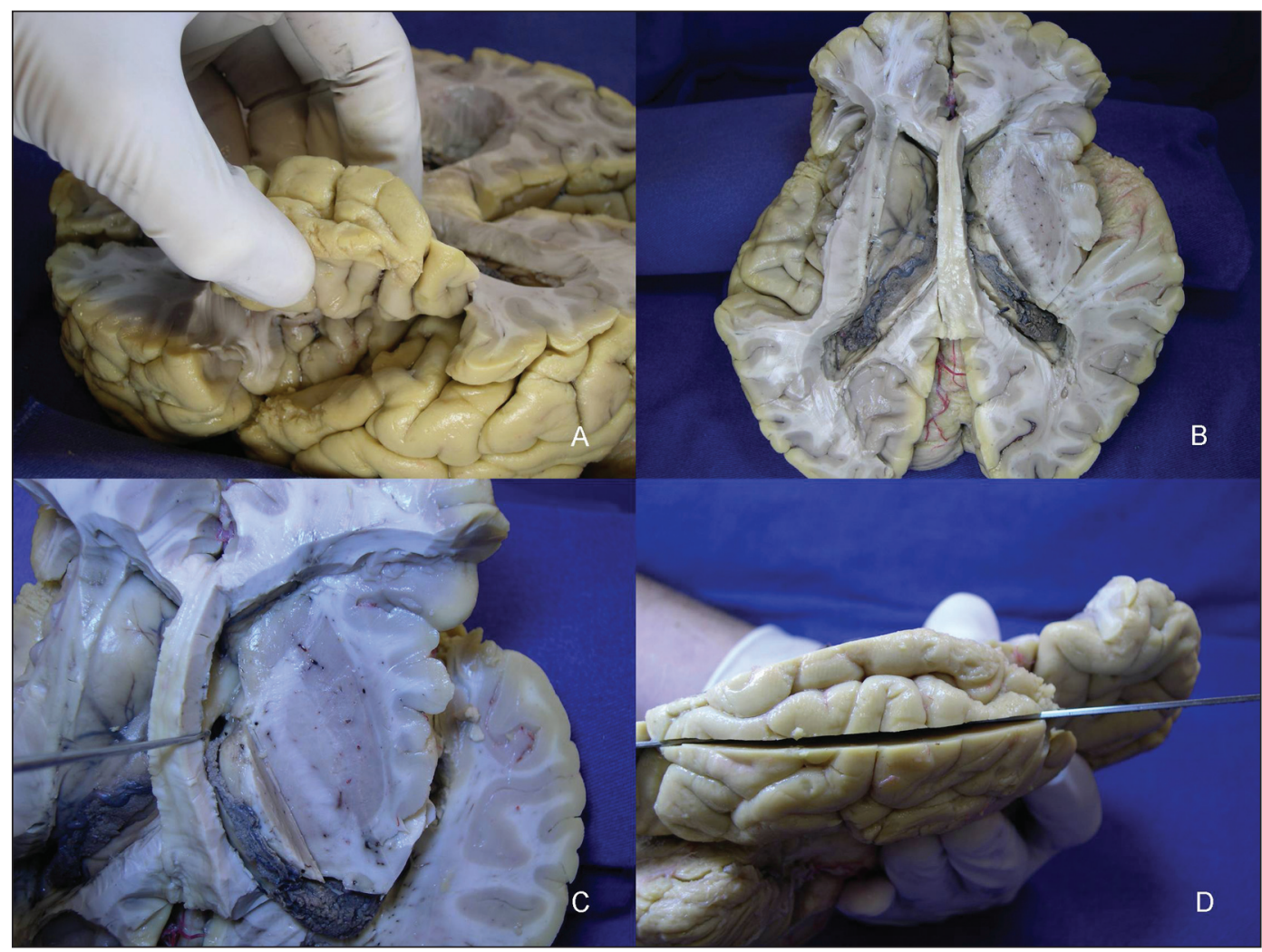

Fig 2. (A) The remaining fronto-partietal operculum must be taken out from the hemisphere to show the insula and the temporal operculum. With one hand, the frontoparietal operculum must be elevated while excised with a number 10 blade along the superior circular sulcus, from the remaining frontal and parietal lobes. The circular sulcus from in sula must be shown in all its length after that, as all the short and long insular gyri as well. (B) At the left side of this specimen, no cuts were made in the insular lobe after the frontoparietal operculum was removed. In the right side, after this step, axial cuts from the superior to the inferior aspect of the insular lobe, the central core of the brain, were made to show the deepest structures in the insular lobe and their relations with the ventricular cavity, frontoparietal and temporal operculum. It's the option A, described in the text. These cuts must be done with the long knife in a lateral to medial way, sparing the thalamus to show the ventricular floor, with the help of a number 10 blade as it gets closer to the ventricles. (C) The foramen of Monro is shown with the dissector and the genu of the internal capsule points it, as a usefull tip seen in MRI images. From lateral to medial, the insular cortex, the extreme capsule, the claustrum, the external capsule, the putamen, the globus pallidus, the internal capsule, the caudate nucleus, the thalamus, the choroid fissure, and the fornix should be exposed after these axial cuts in the central core, as shown in the picture. (D) To expose the temporal horn of the lateral ventricles, a cut with the long knife must be done in the superior temporal sulcus, from lateral to medial, toward the insular cortex, removing the superior temporal gyrus or the temporal operculum. After that, progressively deeper cuts are made from lateral to medial in the temporal lobe pointing the inferior circular sulcus of the insula until the tapetum of the corpus callosum, as the most lateral roof of the temporal horn, is thinned. If it hasn't been opened yet, it can be gently done with the number 10 blade following the same technique used when the anterior ventricular horn, body of the ventricle and atrium was opened. The choroid fissure, the Amon's horn, the collateral eminence, the pes hipoccampus, the thalamus, the fimbriae, the anterior choroidal point and the amigdala in the anterior wall of the temporal horn should be identified after this step, as shown.

terior point is located $2 \mathrm{~cm}$ above the posterior end of the sylvian fissure. The direction of the knife must be followed in direction to the immediately superior part of the corpus callosum to keep its integrity and make the lateral ventricle roof as thin as possible.

The PreC gyrus must be isolated from the remained cortex and put it in its original place to show the relation of this motor area with the corpus callosum and ventricular cavity after its opening (Fig 1C).

After this first cut with the long knife, the medial sur- face of the brain is now clear and even better for identification and study of the medial surface again.

\section{Step 3}

The ventricular cavity must be opened. For this purpose, a number 10 blade is used with careful curved movements to expose the entire ventricle without damage to the insula lobe nor the midline structures. It is described in Figure 1D. The study of ventricular walls, its limits, the relation with the associated structures, the choroid fis- 

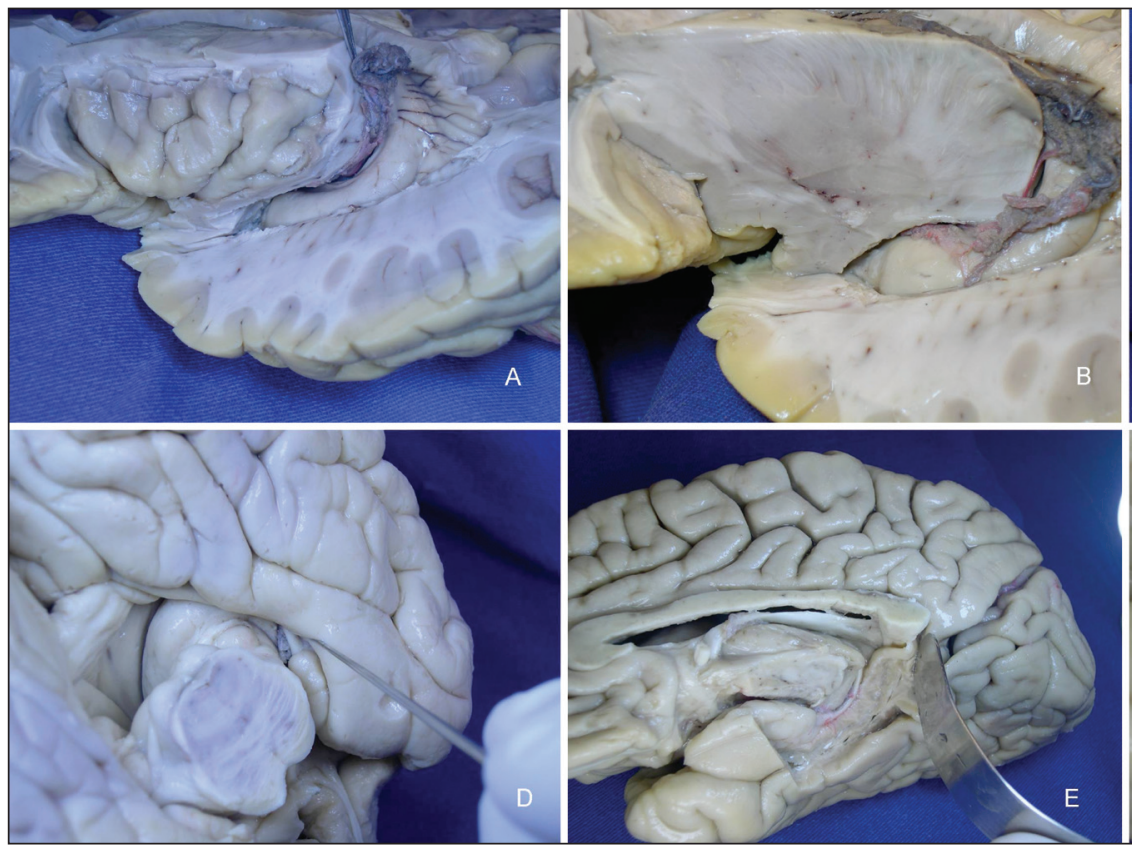

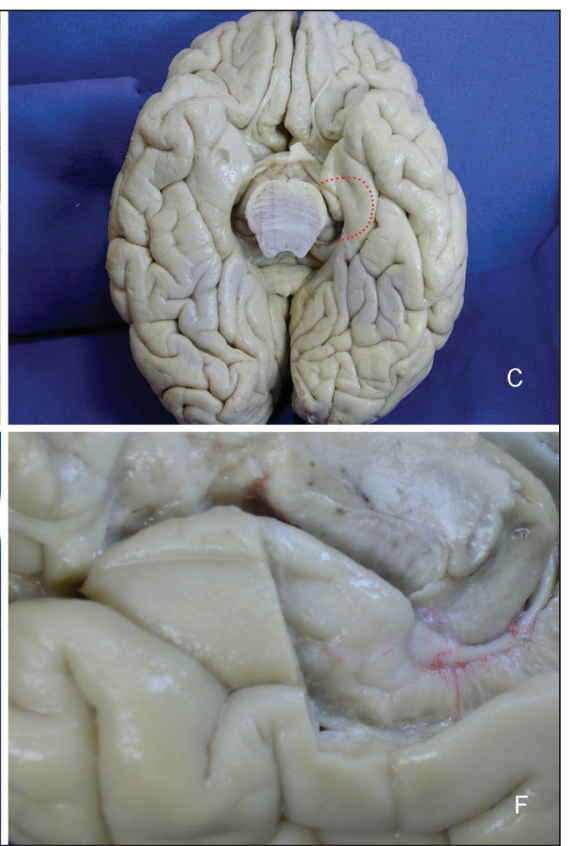

Fig 3. (A) The aspect of the specimen dissected should be as shown in the picture, with the roof of the temporal horn opened by the cuts toward the inferior circular sulcus of the insula. The Ammon's horn, the collateral eminence, the choroid fissure, choroids plexus and the ventricular and atrial walls are exposed, by the dissection. With a number 10 blade, the posterior extension of the temporal horn and atrium should be opened. (B) The option B described in the text is shown in this picture. A sagittal cut was made in the insular lobe. The relations of the ventricular structures with the insular lobe are clear in this dissection. The cuts should be made progressively from lateral to medial, with the long knife, and every cut should be studied before the more medial next one is done. That's because the relations between the structures in the central core are changed in different sagittal planes. The amigdala, located at the anterior wall of the temporal horn, is evident in this picture, and its relations with the globus pallidum, internal capsule and ventricular structures should be done. In these cuts, the different portions of the internal capsule are also evident. (C) Basal surface of the brain. The uncal notch at the uncal apex is used as an initial landmark for the incision in the basal surface of the temporal lobe. This curved incision, shown by the red dotted line in the picture, doesn't have to be made in only one movement. Instead, progressively medial to lateral curve cuts should be made toward the collateral sulcus. The objective is to remove the inferior or basal part of the uncus. There is an arachnoid plane and vessels coming from the crural and ambient cistern in the uncal notch which helps the identification of the correct plane of dissection. Posteriorly, the incision removes the subiculum, making more evident the choroid fissure from below. If the correct plane of the dissection was carried on, the dentate gyrus and fimbriae should be exposed without damage in the posterior part of the temporal lobe. (D) The uncal notch of the right temporal lobe is shown in an inferior and medial angle. The uncal notch at the uncal apex, just anterior to the dissector in the picture, is the point where the incision should begin, and is the correct depth of the incision as well. (E and F) After the removal of the inferior part of the uncus and the subiculum, the dentate gyrus and the fimbria just medial to it should be exposed. The identification of the dentate gyrus is made by the unique aspect of this structure, with transversal stripes, and the arachnoid membrane and vessels between it and the uncus and subiculum, evident in the picture. The fimbria is found in the dissection as a white structure medial and superior to the dentate gyrus from above. The fasciolar gyrus is shown at the $3 E$ figure. The lateral geniculate body and the choroid fissure turns clearly exposed after the removing of the subiculum, showing the relations of the temporal horn and the mesial temporal lobe with the thalamus. The optic tract, as the roof of the crural cistern, is evident. The uncinate gyrus, band of Giacomini and intralimbic gyrus are evident in this specimen, after the dissection described.

sure, foramen of Monro, choroid plexus, ventricular vessels, fornix, and septum pellucidum is done ${ }^{4}$. The study of velum interpositum must be done. The surgical routes to access the third ventricle are another training program to this step ${ }^{13}$. The transventricular access to the quadrigeminal cistern is also studied.

\section{Step 4}

The frontoparietal operculum need to be taken out to expose the insular cortex and the opercular side from the temporal lobe. It is shown in Figure 2A. The circular sulcus from the insula, insular gyri, central sulcus of the insula and its limits must be identified. The relation from the insular region to the ventricular, basal ganglia and different portions of the internal capsule is one of the most important elements to understand the third dimension view of the superficial and deep supratentorial structures $^{2,4,7}$ (Fig 2B).

Option A - A progressive deeper cut in axial plane in the insula lobe can be performed to visualize the relation of the internal capsule, lenticular nucleus, thalamus, caudate nucleus and the foramen of Monro inside the ventricles and the insular cortex. It is described in Figures $2 \mathrm{~B}$ and $2 \mathrm{C}$. 


\section{Step 5}

Exposing the temporal horn of the lateral ventricle and the atrium. The long knife is used to perform the cut taking the superior temporal sulcus as a landmark. The procedure is described in Figure 2D. The opening of the inferior circular sulcus from insula and entering the temporal horn of the lateral ventricle will allow the identification of Ammon's horn, the collateral eminence, the choroid fissure, and the ventricular and atrial walls. The study of the choroid fissure at this level must be carried out to expose the ambient cistern and its content as its relation to the ventricular structures ${ }^{4,5,14}$ (Figs $3 \mathrm{~A}$ and 3B).

Option B - At this level, an option B is available if the option $A$ was not carried out or at the other side of the brain, opposite to the axial sliced central core. A sagittal cut can be done if the intention is to study the amigdala and its relations to the globus palidum, uncus, ventricular roof and anterior ventricular walls and also the identification of the retro and sublenticular parts of the internal capsule. The identification and the study of the choroid fissure is also facilitated from this point of view (Fig 3B).

\section{Step 6}

Mesial temporal lobe dissection. Initially, a curved incision is made in the medial aspect of the parahipocampal gyrus using the apex of the uncus, formed by the ambient gyrus of the uncus, and uncal notch (Figs $3 C$ and 3D) anteriorly and the lateral mesencephalic sulcus posteriorly as landmarks. Progressively deeper and laterally oriented cuts are done toward the rhinal sulcus, with the objective of removing the parahipocampal gyrus and subiculum (Figs 3E and 3F). The dissection plane is assured by the vessels entering the uncal notch from de carotid and crural cistern. After that, the dissection should expose the fimbriae, the dentate gyrus, the fimbrio-dentate sulcus, choroid fissure, the lateral and medial geniculate bodies and pulvinar of the thalamus and the three little gyri of the posterior segment of the uncus (uncinate gyrus, band of Giacomini and intralimbic gyrus). The anatomic relations between these structures, as the relations between them and the thalamus and geniculate bodies, optic tract and mesencephalon, especially the lateral mesencephalic sulcus should be observed ${ }^{2,4,5,14}$.

\section{DISCUSSION}

Since the relation between neuroanatomy and the clinical pictures produced by every structure and their lesions is evident, the study of neuroanatomy, especially of the brain, is essential for many professionals. Most of the articles and books in the subject describes the structures and the relations between then, and do not describe the dissection exercise. We believe that this exercise is a priority and since new specimens are rarely avaiable in most of the laboratories, the dissection and preparation of these specimens are very important, and should be guided with the purpose of showing the largest number of structures as possible, with minimal damage of the piece. For that, a standardized routine sequence of cuts is the best choice. The "step-by-step" dissection described, allow the student to identify all the important structures in each fase of the study, without the risk of damaging other parts of the brain in the attempt to show one in particular. In the end of the study, the student must check the structures studied, as a checklist, to be sure that none of then was left behind.

In conclusion, the dissection is a essential part in the study of neuroanatomy, especially for neurosurgeons, because of the numerous structures in the brain and the clear relation between then and clinical pictures. For that, a sequence of well defined dissection steps assures the identification of all the important structures of the brain, without wasting the specimen.

\section{REFERENCES}

1. http:// mecsrv04.mec.gov.br/acs/asp/noticias/ noticiasId.asp?Id=2274

2. Rhoton AL Jr. The cerebrum. Neurosurgery 2002;51(Suppl 1):S1-S51.

3. Yasargil MG. Microneurosurgery, Vol I. Stuttgart: Georg Thieme Verlag, 1994:5-168.

4. Rhoton AL Jr. The lateral and third ventricles. Neurosurgery 2002; 51(Suppl 1):S159-S201.

5. Wen HT, Rhoton AL Jr, Albert L Jr, et al. Microsurgical anatomy of the temporal lobe: Part 1. Mesial temporal lobe anatomy and its vascular relationships as applied for amygdalohippocampectomy. Neurosurgery 1999;45:549-592.

6. Rhoton AL Jr. Cranial anatomy and surgical approaches. Neurosurgery 2003;53:1-746.

7. Ribas GC, de Oliveira E. A ínsula e o conceito de bloco cerebral central. Arq Neuropsiquiatr 2007;65:92-100.

8. Ribas GC, Ribas EC, Rodrigues CJ. The anterior sylvian point and the suprasylvian operculum. Neurosurg Focus 2005;18:E2.

9. Ribas GC. Pontos referenciais nos acessos cranianos. Arq Neuropsiquiatr 2003;61:1071-1072.

10. Ribas GC, Yasuda A, Ribas EC, Nishikuni K, Rodrigues AJ Jr. Surgical anatomy of microneurosurgical sulcal key points. Neurosurgery 2006; 59(Suppl 2):S177-S208.

11. Gusmão S, Reis C, Tazinaffo U, Mendonça C, Silveira RL. Definição do limite anterolateral do lobo occiptal em peças anatômicas e exames de imagem. Arq Neuropsiquiatr 2002;60:41-46.

12. Martins C, Yasuda A, Campero A, Rhoton AL Jr. Microsurgical anatomy of the oculomotor cistern. Neurosurgery 2006;58(Suppl 2):S220-S228.

13. Wen HT, Rhoton AL Jr, de Oliveira EP. Transchoroidal approach to the third ventricle: an anatomic study of the choroidal fissure and its clinical application. Neurosurgery 1998;42:1205-1219.

14. Campero A, Tróccoli G, Martins C, Fernandez-Miranda JC, Yasuda A, Rhoton AL Jr. Microsurgical approaches to the medial temporal region: an anatomical study. Neurosurgery 2006;59(Suppl 2):S279-S308. 